\title{
Json is Efficient over the XML in Native Application
}

\author{
Katte Darshan A. \\ M.Tech (IT), Student \\ Vellore Institute of Technology, \\ Vellore, India
}

\author{
Suganya P. \\ Assistant Professor, \\ Vellore Institute of Technology, \\ Vellore
}

\begin{abstract}
Json is broadly known as one of the restless Service. Its standard for communicating the data objects is, the Key-Value pair format. The above cited restless service largely adopted allochronic forms with the browser and server. Currently it's used by AJAX. In rest services, a request can be identified by a particular request to URL, which gives responses in XML, JSON or HTML. This is possible since rest services are able to cope up with large data processing tasks. Simulation of Json and XML output based web service was carried out. The simulation results helps out to choose better communication service in the native apps. With the help of simulation results, we observe that, Json can largely be adopted over the web application, it being very thin object which can be communicated and conveyed to any native application.
\end{abstract}

\section{Keywords}

Json (JavaScript Object Notation), AJAX (Asynchronous JavaScript and XML), XML, HTML, NATIVE.

\section{INTRODUCTION}

As Json is one of the Open Grade protocol which extends the better use or exist from the JavaScript Programming Language. With the insubstantial decompose facility. Also it can used to maintain the overall status log of activities and thus it can be used in the real time client and the server form .and it supports http.thatswhy it is widely known as intercourse self-determined writing code. Script for transmitting and creating Json text which is readily available in any platforms suitable data constructs. Also Json has the merits like reconcilability and the forthrightness. Web administration and application programs utilize Json organization to give open information.

JSON is configured on bellows two form

A] An accumulation of tag value pair. And In different dialects this is acknowledged as a struct, word reference, hash table or acquainted exhibit.

B] A requested rundown qualities. In many dialects, this is acknowledged as an exhibit rundown or succession.

Now in current world, offbeat stacking of information is critical. As such, site pages need to have the capacity to stack new information without refreshing the whole page to get it. This outcomes in a smoother and more ideal perusing background. JSON is incredible for sending and accepting non concurrent information since its basic and simple to utilize.

Xml which obtained from GGML (Grade Generalize Mark Lang).Also its one of the sentence included with selfdetermining capability. The XML linguistic structure utilizes coordinating begin and end labels, (for example, $\langle$ tag $\rangle$ and $</$ tag) to increase data. Data delimited by labels is called a component. Each XML archive has a solitary root component, which is the top-level component that contains the various components. Components that are contained by different components are regularly alluded to as sub-components. A component can alternatively have characteristics, organized as name-esteem combines that are a piece of the component and are utilized to additionally characterize it. Xml stores normal sentences and their related data and it sends the data and maintain and creates updated data and sends and retrieves the related data.

Below Is the Example Of Json the main difference between the $\mathrm{xml}$ and json is the tags It's easy to transfer the Json data and does not needs to create the tag because Each tag in $\mathrm{xml}$ needs to create its end tag.

In the Example of Json it needs to create with tag and value pair. Because its having own key and value like Name is the key for the address list is value pair .and Address is the key pair for the list address od person name1 and its having the list of attributes like Name, LastName, Street, Area ,City.

Example of Json

\{

Name: \{

Address : \{

Address of Person Name1 : \{

Name: "XYZ",

LastName: "ABC",

Street: " $5^{\text {th }}$ cross Lawrence Layout",

Area: "Veeranapalya"

City: "Bangalore "

\}

\} Name: XYZ

Here are the some Merits over the Xml

A] As json contains the structure of correct information and $\mathrm{xml}$ is one of the organizational structure In spite of the fact that a guide (simply key/esteem sets) can point of confinement that is the thing that we need, since it is less demanding to decipher and is unsurprising.

B] Json is demonstrated as its one of the restricted .some may think like xml is good but the alignment of json is better than $\mathrm{xml}$ because $\mathrm{xml}$ stores the data in one of the structured way.

In the below everything information is stored in open and close tag of Address and each statement needs the opening and closing tags in $\mathrm{xml}$. 


\section{Example of Xml}

<Address>

$<$ Name $>\mathrm{XYZ}<$ /Name $>$

$<$ LastName $>\mathrm{ABC}</$ LastName $>$

$<$ Street $>5^{\text {th }}$ cross Lawrence Layout $</$ Street $>$

$<$ Area $>$ Veeranapalya</Area $>$

$<$ City $>$ Bangalore $</$ City $>$

$</$ Address $>$

Json is insubstantial thin data which is very easy to understand the one by one line understanding of structured data. As Json contains different data like string, array, list but $\mathrm{xml}$ is transferred in one of these or all of the standards.

So Json data can be used to transfer the data over the internet to one of the native apps which can be used to build iOS apps .Such as native structure can be chosen for the specific things so these things will take particular advantage of limitation and it will be one of the great security while defining the intent filters between theses IOS native apps.

So native apps different platform to integrate the data from the base system Such as here in these paper so Full native apps uses web services as the correct exchange of data into the app So on that base sql server to store the database table swathe specific stored procedures upon the built trigger to specify the particular action web service use the stored procedure to provide as input and web services pulls the data from sql and app integration code is written to integrate the data with the app.

\section{LITERATURE SURVEY}

According to Tommy Aihkisalo and Tumaas Paaso tiled "Latencies of Processing of REST and SOAP Web service Interface" [1] in this paper they compared one message. I.e. Message can be anything like video, audio and raw data. They sent video from the sender to particular requester who requested the video. Got the result of the time when the hit occurred that is request time for the video and average waiting time to load an instance of a video.

They calculated the time to load an instance on webpage .compared both the result with rest and SOAP and given conclusion on that and shown the differences in XML and JSON also.

In the paper tiled as "Improving Data transfer in web applications via translation between $\mathrm{xml}$ and the Json" [2] written by Guanhua wang according to him produced the test report in the serialized approach while transferring the data over the network in web application .they proved by the test result like $\mathrm{xml}$ is one of secure thing for transferring the data over the web application and Json can convenient for the fast data transfer and one of convenient approach for serializing the data in the web applications.

In order to use third level web protocols they opted in applications as the xml but for the serialization purpose Json can be one of the convenient approach for the speedy recovery of data according the Serialized methods. But in the AJAX approach as ensuring that they will give good enhancement with experience. Keeping this in mind Json will be perfect approach for the recovery of data but the in order to make safe communication via secure way then they opted the Xml for secure data transfer in the third party web application which transfer the data via third party translator. Here both XML and JSON considered as the Hierarchical structure as the JSON having the key and its related value pair. Same repeated tags are removed from the xmls.

According to Boci Len, Yan Chen, Xu Chen's tiled paper [3]"Comparisons of JSON and XML in AJAX "they proved that Json is better than $\mathrm{xml}$ in the new web application of AJAX,As the main Severe Reason is as $\mathrm{xml}$ is traditional old language needs to restricted at both the client and server side this causes one of the drawback and the main thing is JSON is thin thing to pass data across the network and it's very easy and convenient way to transfer the data as early as possible.

Here they calculated the efficiency in both the sides i.e. server side and client side in terms data sending with the help of $\mathrm{xml}$ ,same with the help of the JSON as the compared results suggests that JSON performs well over the AJAX wee applications. As xml is based on document protocol it needs to decode at the both the ends. And JSON has the capability of serializing the objects both at the client side and the server side this is the main reason due to that the corresponding webpage retrieved sooner. Also they measures client transfer capability end of the xml and JSON and given the results with the best convenient way of data transfer is JSON in AJAX.

In the paper tiled as "Improving the efficiency of presence service in IMS by JSON" [4] written by Kun-Che HSU, JenqLeu.in this paper as the current trend is changing over voice over the IP data .So IP based multimedia has great potential to provide the audio so they proved like $\mathrm{xml}$ can be used to detect the presence of particular thing and json can be used for data sending through one another as its very thing to pass the data over the network. And instead of using xml they used Json and proved which is like $54 \%$ overhead is reduced with the help of JSON transfer of data over the network. So they built one IM standard phenomena based on that they compared the their average waiting time of the $\mathrm{xml}$ and the Json over each other and shown the json waiting time is less as compared to XML.

So anyone who is using mobile they need to subscribe through the WEBRTC and built the gateway point at the both end checked the time to live of Data from both xml and json. Here the IM standard used the Sip data which was duplicated over the IM standard network.

They show SIP data duplicate has been used webrtcbreaker placed in the different Im standard which proves the presence of $\mathrm{xml}$ was heavy in terms Json as json sends the data in the tag and related data pair it becomes very thin to pass the data over the communication link. As xml becomes the heavy presence at the both end of the data in this architecture they used different media transfer file which can be used to encode and decode the specific audio and video data in the transmission of particular instance over the Im standard network.

\section{RESULTS AND COMPARISON}

\subsection{Json Results}

The result consists of load time to create or load the web service data onto the particular machine and also it includes the test data for to load $\mathrm{xml}$ data below are the comparison results. 


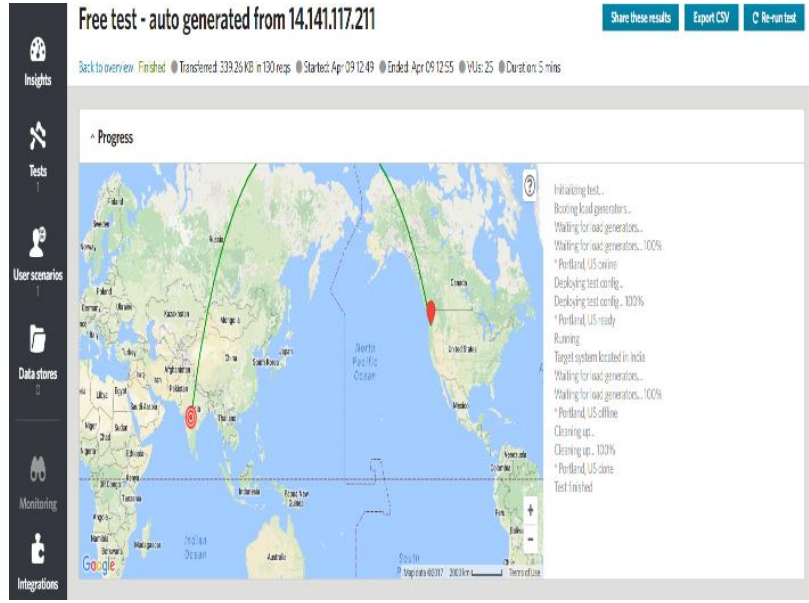

Fig (A) Json Data Mapping

The above figure contains the particular data needs to download particular request and it contains no of bits to download and transfer the particular data also it contain the no virtual users requested the particular page. With the start time and the end time indicated in the result.

The bellow's main chart indicates the no virtual users are active on the particular site and the time required to load on particular screen in their mobiles native app here these are the minimum time to load data in the native app.The load time is very minimal for loading the json web service into native app so the green line indicates the load time and that ratio below the green line indicates the fetching time of particular page from the web pool. And faint blue line indicates the no of virtual users are active so while parsing json data and loading the data it's very easy to send and receive over the network because load time is very less. And the chart shows the ratio of $\mathrm{VU}$ active and time to load the data.

Mainchart

OWsatie OWlard tin

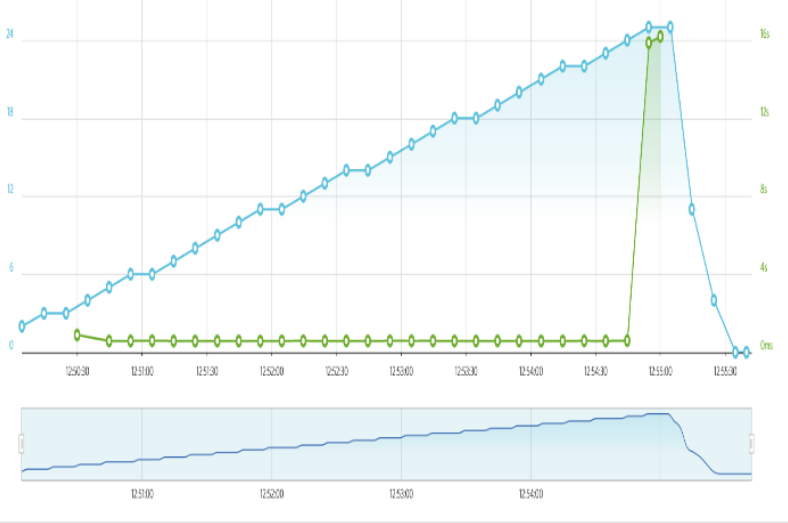

Fig (B) Main chart of Virtual user Load time

The below $\operatorname{fig}(\mathrm{C})$ contains the time to load the site that can consumes and the second graph indicates the no of requests came up for the seconds. And shows which tcp connections it needs to enable. Also it shows the different for loading the page from memory and how much it consumes memory when it's loaded on the specific page. Last one indicated the load time of the particular page.

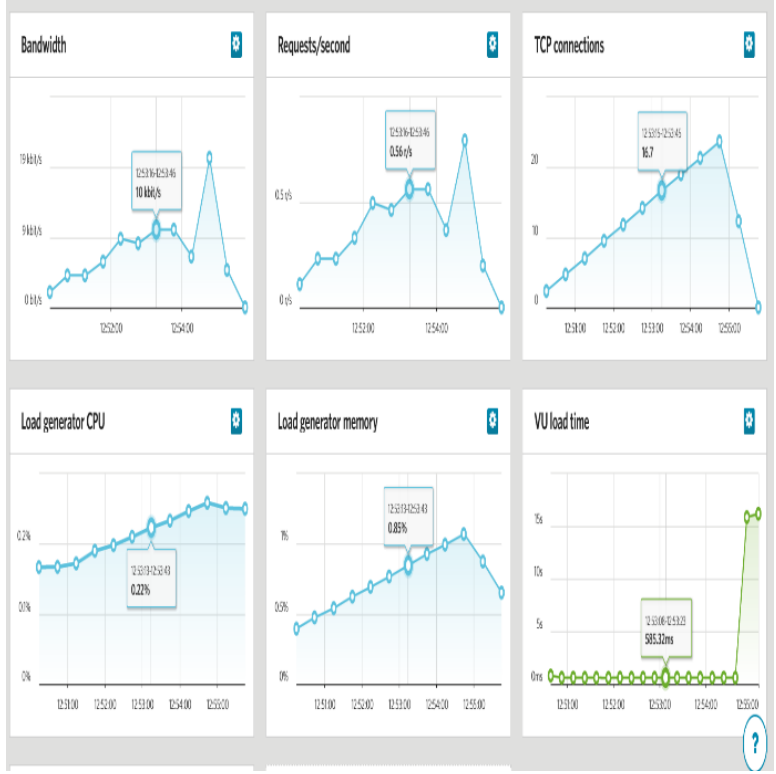

$\operatorname{Fig}(C)$ all the symptoms chart to load the basic data.

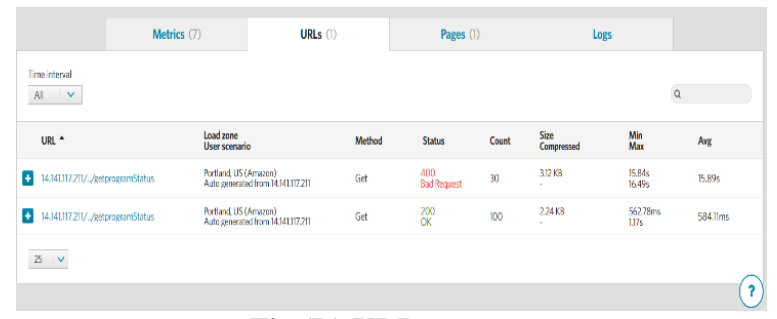

Fig (D) URL request.

The above figure contains the type of URL which it has with the type request that is get request.

\subsection{XML Results}

Now all the graphs which occurs now is the data for the XML data and its related charts with the load times and failure times.

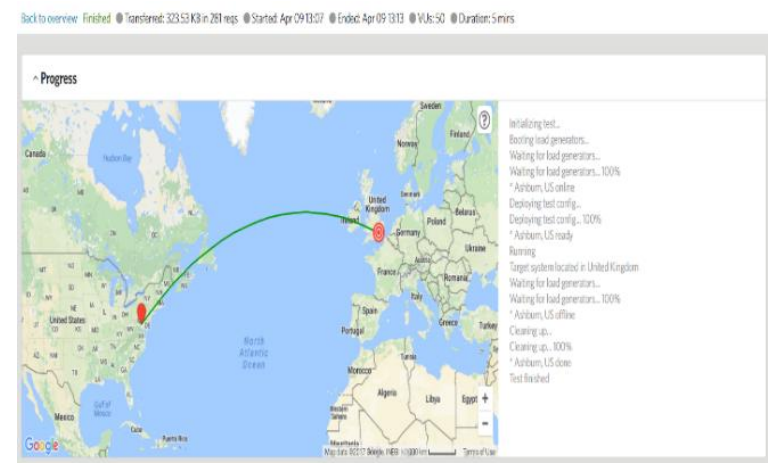

Fig (E) XML Data Mapping

The above graph contains the $x m l$ parsed data on to the particular site so it will retrieve the total time required to download the particular xml tagged data it show downloaded data in no of bytes .also it shows the no of active users on that $\mathrm{xml}$ site .and the mapping refreshing of the data from the different regions and also it shows the no virtual users are active on the particular site.it shows test starting and ending time now comparing the both results like xml and json Fig(e.xml) and Fig(a) . Json data mapping while parsing both the data form these two graph we only get the amount of the transfer time but rest all thing are same compared to both. 


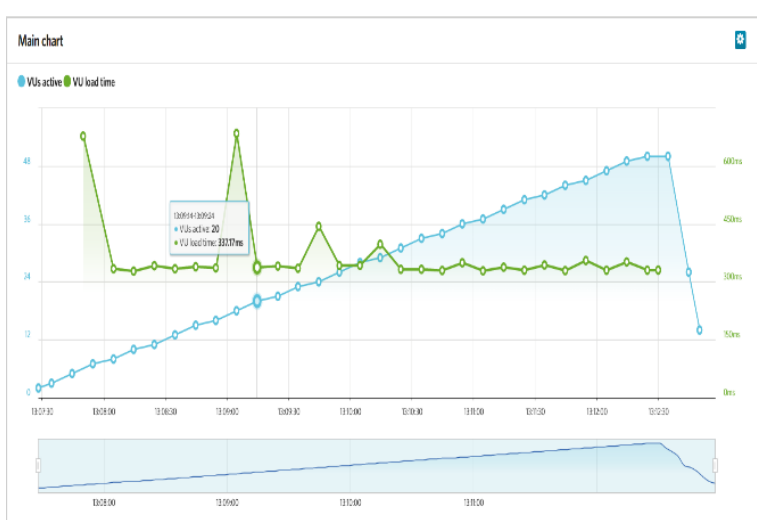

Fig (F) Main chart of virtual user load time of xml data mapping

By comparing fig (B) and the fig (f.xml) we will come to the final opinion like virtual load time of json is less than the Virtual load time of the xml. Actually with the xml even the load time is increased more than the no of users active in that particular session so it's one of the big drawback xml over the json while parsing the data thatswhy Json is very thin insubstantial thing to interpret the line by line in a structured manner so the same thing which gives conclusion like json data is very efficient in native apps because it boosts performance by making use of efficient structure and its related data over the network.

Also Json can be used with various data type this feature makes json very easy and portable thing.so that it can easily transfer the data from the ne end to another end via native app structure one of the prime advantage of json in Native apps the above one and it's very easy parse and keep the data in the structured protocol. And it can be persevered and updated according to the requirements.

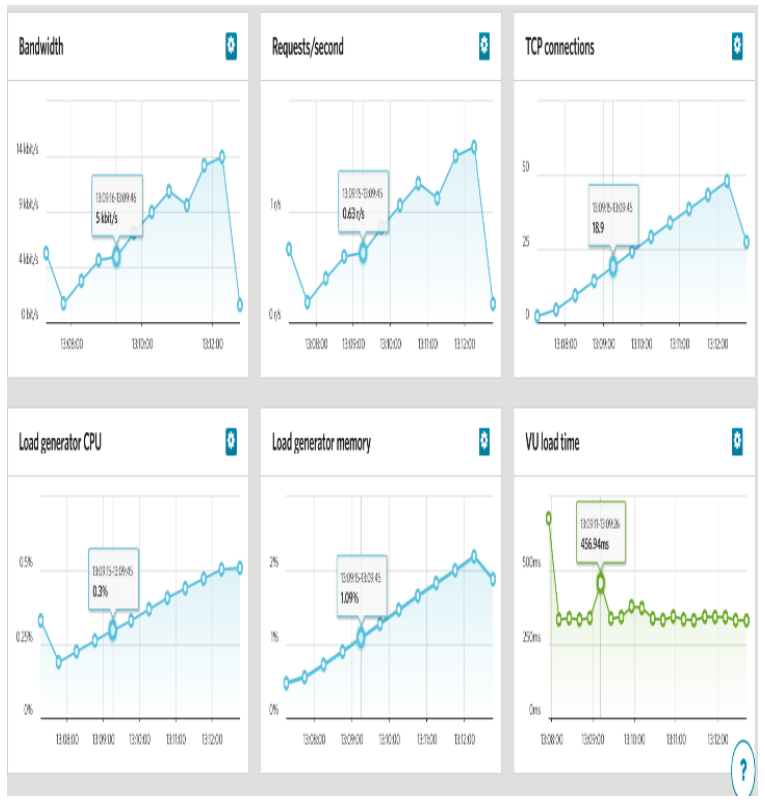

Fig (G) All Symptoms data to load the particular Xml data

By comparing fig(c) and fig (g.xml) we still get a clarity of efficiency of json in terms of bandwidth. So in order to calculate the bandwidth it requires the first instance to load the data and the last instance to last the data the gap between the two instance can be the bandwidth according to these graphs the memory consumed by $\mathrm{Xml}$ is more the Json because after loading the page its load . also it can be a drawback to parse the large amount of data it's become one of the challenge here above took small example proved the load time of json is less and also very easy to send the data in different format i.e. iOS native apps.

Bellows fig (h.xml) indicates the failure rate to download the particular xml page from the pool so the graph shows the ratio with the start time and the failure.

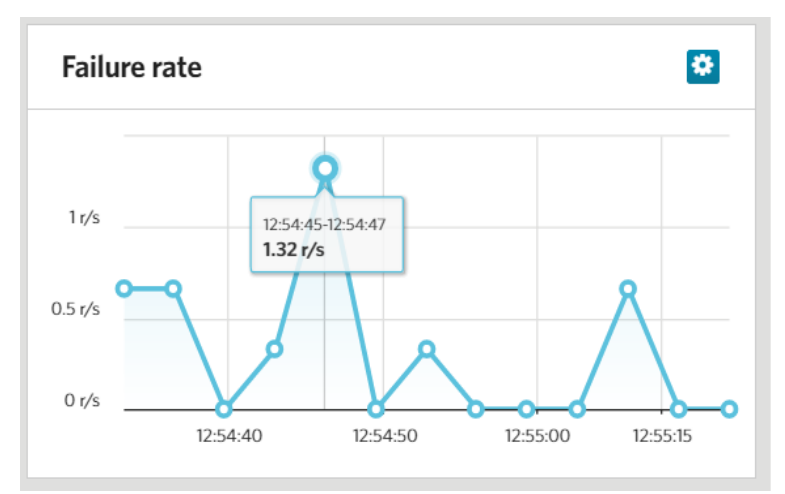

Fig (H) Failure rate of XML

\section{CONCLUSION}

From this paper we conclude that with the help of load testing that is impact of web service in the native web applications can be compared with virtual load for the particular web service i.e. XML or JSON by using $\mathrm{xml}$ it can be secure .But very easy and fast way of encoding is JSON as load time to load particular web service of JSON is less than of the XML and failure rate also very less Compared to XML.

\section{ACKNOWLEDGMENTS}

I would like to express my special gratitude and thanks to Prof. P.Suganya, Assistant Professor, School of Information Technology and Engineering whose esteemed guidance and immense support encouraged to complete the work successfully.

\section{REFERENCES}

[1] Aihkisalo, T., \& Paaso, T. (2012, June). Latencies of service invocation and processing of the rest and soap web service interfaces. In Services (SERVICES), 2012 IEEE Eighth World Congress on (pp. 100-107). IEEE.

[2] Wang, G. (2011, April). Improving data transmission in web applications via the translation between XML and JSON. In Communications and Mobile Computing (CMC), 2011 Third International Conference on (pp. 182-185). IEEE.

[3] Lin, B., Chen, Y., Chen, X., \& Yu, Y. (2012, August). Comparison between JSON and XML in Applications Based on AJAX. In Computer Science \& Service System (CSSS), 2012 International Conference on (pp. 11741177). IEEE.

[4] Hsu, K. C., \& Leu, J. S. (2015, July). Improving the efficiency of presence service in IMS by JSON. In Ubiquitous and Future Networks (ICUFN), 2015 Seventh International Conference on (pp. 547-550). IEEE. 